\title{
La sphère, représentation du ciel et de la terre : une histoire pluridisciplinaire
}

L'exposition Le monde en sphères, Bibliothèque nationale de France/site François Mitterrand

\section{Catherine Cardinal}

\section{(2) OpenEdition} Journals

Édition électronique

URL : http://journals.openedition.org/artefact/4227

DOI : $10.4000 /$ artefact. 4227

ISSN : 2606-9245

Éditeur :

Association Artefact. Techniques histoire et sciences humaines, Presses universitaires du Midi

Édition imprimée

Date de publication : 15 octobre 2019

Pagination : 201-205

ISBN : 978-2-8107-0650-1

ISSN : 2273-0753

Référence électronique

Catherine Cardinal, «La sphère, représentation du ciel et de la terre : une histoire pluridisciplinaire », Artefact [En ligne], 10 | 2019, mis en ligne le 06 août 2020, consulté le 27 novembre 2020. URL : http:// journals.openedition.org/artefact/4227 ; DOI : https://doi.org/10.4000/artefact.4227

\section{(c) (i) (2) $\Theta$}

Artefact, Techniques, histoire et sciences humaines est mise à disposition selon les termes de la Licence Creative Commons Attribution - Pas d'Utilisation Commerciale - Pas de Modification 4.0 International. 


\section{La sphère, représentation du ciel et de la terre : une histoire pluridisciplinaire}

\section{L'exposition Le monde en sphères, Bibliothèque nationale}

de France/site François Mitterrand

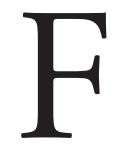

ondée sur l'histoire des représentations sphériques du ciel et de la terre, la thématique de l'exposition est élargie à de nombreux domaines propices à des réflexions variées et illustrés par des œuvres très diverses. Sciences, techniques, archéologie, philosophie, arts concourent à disséquer l'histoire de la vision sphérique du monde depuis 2500 ans. L'évolution du globe comme image de l'univers terrestre et céleste est mise en lien non seulement avec les découvertes scientifiques qui la sous-tendent mais aussi, par exemple, avec la curiosité scientifique, la pédagogie, le symbolisme, l'imprimerie, la numismatique.

\section{Un rassemblement inédit de pièces rares}

L'exposition révèle la richesse des collections françaises dans le domaine des sciences et des techniques ${ }^{1}$. Plusieurs pièces mises en valeur, le temps de la manifestation, trouvent la place historique qu'elles n'ont ordinairement pas dans leur lieu de conservation.

1. Exposition présentée au Louvre Abu Dhabi au printemps 2018, puis à la $\mathrm{BnF}$ du 16 avril au 21 juillet 2019. Les commissaires en sont Catherine Hofmann (BnF, département des Cartes et Plans), François Narowcki (bibliothèque Sainte-Geneviève), Jean-Yves Sarrazin ( $\dagger, \mathrm{BnF})$.

95 Catherine Cardinal « La sphère, représentation du ciel et de la terre : une histoire pluridisciplinaire », Artefact, 10, 2019, p. 201-205. 
Seulement une dizaine de globes islamiques antérieurs au $\mathrm{Xv}^{\mathrm{e}}$ siècle, montrant les étoiles et constellations décrites par Ptolémée, est connue. Notons que le musée du Louvre conserve un globe céleste persan daté de 1144/1445, considéré comme le troisième plus ancien globe de cette série (MAO 824), et un globe iranien daté vers 1310/1315 (MAO 825). Quant au musée national de la Renaissance, il possède le plus ancien grand globe céleste occidental conservé, daté de 1502, mesurant $69 \mathrm{~cm}$ de diamètre ; en métal gravé, il présente le positionnement de 750 étoiles (inv. E. Cl. 3218) $)^{2}$.

L'importance des collections du département des Cartes et Plans de la BnF, dans le domaine de la cosmologie et des mathématiques, est mise en évidence par la présence de nombreuses œuvres. Remarquons le globe céleste attribué à Ibn Said al-Sahli, actif en Espagne, au XI ${ }^{\mathrm{e}}$ siècle, le globe terrestre dit "Globe vert " peint sur papier, daté vers 1506, attribué à Martin Waldseemüller. Parmi les pièces les plus spectaculaires de la BnF, figurent la sphère armillaire mouvante montée sur un trépied en bois doré, réalisée entre 1709 et 1718 par l'horloger du roi Jérôme Martinot (Fig. X et XI, cahier couleur), et le monumental globe de parquet ayant servi à l'éducation de Louis de France, daté 1786-1788 caractérisé par un globe terrestre qui s'ouvre pour découvrir, à son revers, la voûte céleste et un globe géologique en relief (Fig. XII, cahier couleur) ${ }^{3}$.

Complétant cette réunion d'objets exceptionnels, dans laquelle on remarque aussi le globe mécanique de John Reinhold ${ }^{4}$, conservé au Musée des arts et métiers (inv. 7491), apparaît la sphère céleste en argent gravé, datée du II $^{\mathrm{e}}$ siècle avant J.-C., issue de la collection Kugel (Fig. XIII, cahier couleur), considérée comme la plus ancienne connue .

2. Destombes, 1968, III, p. 73-81.

3. La plupart de ces pièces ont une notice détaillée dans le catalogue de l'exposition, Hofmann, Narowcki, 2019. L'album édité en parallèle est un utile complément. Se référer aussi à Turner, 2018, p. 81-109.

4. Le globe de Reinhold, daté 1588, offre des analogies avec un autre globe mécanique céleste conservé au Musée des arts et métiers, réalisé en 1582-1583 par Jost Burgi (inv. 7490). Voir leur description détaillée dans Cardinal, Vingtain, 1998, p. 74-76.

5. Rappelons le catalogue de l'exposition, réunissant une quarantaine de globes et de sphères armillaires, par Kugel, 2002. A propos de ce globe, voir p. 22-27. 


\section{Un parcours chronologique diversement documenté}

Les origines de l'image sphérique du monde céleste et de l'univers terrestre sont d'abord précisées ${ }^{6}$. Elles remontent aux recherches de Pythagore au $\mathrm{VI}^{\mathrm{e}}$ siècle avant J.-C., suivies par celles de Platon et Aristote. Ce modèle matérialisé par des sphères concentriques, portant les étoiles et les planètes, tournant autour de la sphère terrestre immobile, est perfectionné par Ptolémée au $\mathrm{II}^{\mathrm{e}}$ siècle. Une suite de manuscrits enluminés témoigne ici des recherches d'Aristote et des travaux du cosmographe, notamment une copie de son Almageste datée vers 1170-1200. Une deuxième étape fait ressortir la transmission de la conception antique au monde arabo-musulman puis, à partir du XI siècle, à l'Occident. Dans ce contexte, l'exposition des rares globes du Louvre et de la BnF, fabriqués selon les données de l'Almageste, prennent tout leur sens. Au $\mathrm{Xv}^{\mathrm{e}}$ siècle, les voyages d'exploration permettant de préciser la géographie de la terre, les premiers globes terrestres sont réalisés. Le " Globe vert " est le premier conservé à représenter le Nouveau Monde.

Sous la Renaissance, l'héliocentrisme formulé par Copernic remet en question la représentation géocentrique de l'univers. Les sphères armillaires, qu'elles soient géocentrées ou héliocentrées, et les globes terrestres sont produits en nombre croissant durant le XvII ${ }^{\mathrm{e}}$ siècle, notamment à la demande des curieux ou des érudits. Les paires de globes, l'un terrestre et l'autre céleste, imprimés sur papier, fabriqués dans des formats de 10 à $70 \mathrm{~cm}$, sont à la mode. Les fabricants hollandais comme les familles Hondius, Blaeu et van Langren acquièrent une renommée dans cette spécialité comme le démontre une abondante série de globes regroupés pour l'occasion.

Au XVIII ${ }^{\mathrm{e}}$ siècle, la production de globes à l'usage des amateurs de science se développe à Paris. Ceux du savant abbé Nollet, comme la paire réalisée vers 1728-1730 appartenant à la $\mathrm{BnF}$, sont représentatifs de cette vogue répandue dans les salons. Les globes et les sphères armillaires deviennent des objets indispensables à l'éducation. Trois portraits ont été réunis pour

6. Il est à noter que le catalogue de l'exposition, divisé en cinq grandes parties chronologiques, reprend le plan rigoureux et clair de la présentation muséographique. L'ouvrage fait judicieusement alterner des essais, des descriptions d'" œuvres phares » et des focus. 
témoigner de cet usage : le premier, par François de Troy, daté de 1705, représente La Leçon d'astronomie de la duchesse du Maine au château de Sceaux; le deuxième, par Louis Tocqué, daté de 1739, montre le dauphin Louis de France dans son cabinet de travail ; le troisième, par Anne-Louis Girodet-Trioson, daté 1803, dit La leçon de géographie, réunit dans un têteà-tête studieux le docteur Trioson et son fils Benoît ${ }^{7}$.

Les aspects symboliques du globe ne manquent pas d'être soulignés ${ }^{8}$. Image de la puissance des empereurs romains et des rois, il figure sur maints supports, médailles, pièces de monnaie, gravures, peintures, etc. Le camée dit le "Triomphe de Licinius " montrant l'empereur sur son quadrige, tenant un globe céleste et une lance, en train d'écraser ses ennemis est une représentation éloquente de cet attribut ${ }^{9}$. Allégorie de la vanité, le globe est souvent associé aux figures de la Fortune, du Temps et de la Mort. La nature morte attribuée à Jan Davidz de Heem réunissant un crâne, une montre, des partitions éparpillées et un violon abandonné auprès d'un globe, avec la formule Vanitas vanitatum et omnia vanitas inscrite sur la carte qui pend sur le mur, offre le parfait exemple d'un memento-mori ${ }^{10}$.

204 À l'époque contemporaine, les sphères armillaires sont abandonnées au profit des planétaires. En revanche, outils indispensables de la pédagogie, les globes terrestres se répandent, dans des dimensions et des techniques variées. S'appuyant sur l'actualité scientifique, la section finale évoque combien la terre est minuscule et fragile, bien loin de son image conçue au centre d'un univers clos par les savants de l'Antiquité.

La paire de globes gigantesques offerte à Louis XIV en 1683 par le cardinal d'Estrées, réalisés à Paris sous la direction du moine vénitien Vicenzo Coronelli, enrichit assurément l'exposition qui fait écho à leur présentation dans le hall Ouest de la $\mathrm{BnF}^{11}$.

Catherine Cardinal Université Clermont-Auvergne

\footnotetext{
7. Musée du domaine départemental du château, Sceaux, inv. 88.24.1 ; musée national des châteaux de Versailles et Trianon, MV 3789 (copie) ; Montargis, musée Girodet, inv. 005.1.1

8. À compléter par Hofmann, Lecoq, Netchine, Pelletier et al., 1995.

9. BnF, département des Monnaies, médailles et antiques, Camée 308.

10. Musée des beaux-arts de Rouen, inv. 1907, 1,125.

11. Hofmann, Richard, 2012 et Richard, 2006.
} 


\section{Bibliographie}

Cardinal Catherine, Vingtain Dominique (dir.), Trésors d'horlogerie. Le Temps et sa mesure du Moyen Âge à la Renaissance, RMG/Palais des papes, Avignon, 1998.

Destombes Marcel, «Un globe céleste inédit de l’année 1502 », Actes du XI congrès international d'histoire des sciences, Ossolineum, collection des travaux de l'Académie internationale d'histoire des sciences, Wroclaw, 1968, p. 73-81.

Hofmann Catherine, Lecoq Danielle, Netchine Ève, Pelletier Monique, Le globe et son image, BnF Éditions, Paris, 1995.

Hofmann Catherine, Richard Hélène (dir.), Les Globes de Louis XIV. Étude artistique, historique et matérielle, BnF Éditions, Paris, 2012.

Hofmann Catherine, Nawrocki François (dir.), Le monde en sphères, BnF Éditions, Paris, 2019.

Kugel Alexis, Sphères. L'art des mécaniques célestes, J. Kugel, Paris, 2002.

Richard Hélène, Les Globes de Coronelli, BnF Éditions/Seuil, Paris, 2006.

Turner Anthony, Mathematical Instruments in the Collections of the Bibliotheque nationale de France, BnF Éditions/Brepols, Paris, 2018. 